\title{
Knowledge, Perception, Attitudes and Behavior on Influenza Immunization and the Determinants of Vaccination
} \author{
Nisreen Sidani ${ }^{6}$, Mohamad Yasminn, (1), Ali Rteil ${ }^{6}$, Souha S. Kanj ${ }^{6}$, Zeina A. Kanafani ${ }^{6}{ }^{*}$ \\ ${ }^{1}$ Department of Internal Medicine, University of Toledo Medical Center, Toledo, OH, USA \\ ${ }^{2}$ Department of Internal Medicine, Mckeesport Internal Medicine Center, Pittsburgh, PA, USA \\ ${ }^{3}$ Department of Biostatistics and Epidemiology, University of Cambridge, UK \\ ${ }^{4}$ Department of Internal Medicine, Case Western Reserve University, Cleveland, OH, USA \\ ${ }^{5}$ Department of Internal Medicine, Cleveland Clinic, Cleveland, OH, USA \\ ${ }^{6}$ Department of Surgery, American University of Beirut, Beirut, Lebanon
}

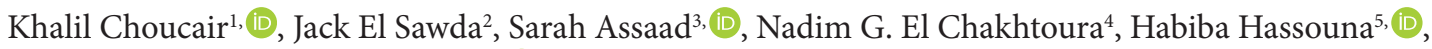

\section{ARTICLE INFO}

\section{Article History}

Received 24 September 2019

Accepted 22 August 2020

\section{Keywords}

Influenza

determinants of vaccination

healthcare workers

health-belief model

$\mathrm{KAB}$

perception

\begin{abstract}
Background: We sought to determine the knowledge of, perception, attitudes, and behaviors toward influenza virus and immunization, and the determinants of vaccination among students, patients, and Healthcare Workers (HCWs) at the American University of Beirut and its affiliated Medical Center.

Methods: We conducted a cross-sectional study between October 2016 and January 2017 utilizing a self-administered questionnaire that was provided to 247 randomly selected adult participants. Data collected included socio-demographic characteristics, prior vaccination against influenza, knowledge, perception, attitudes, and behaviors toward influenza and influenza immunization. A multivariable regression model was used to evaluate for independent associations between the different variables and regular or yearly vaccination as a primary outcome.

Results: The overall survey response rate was $77 \%$. A substantial proportion of respondents $(47.4 \%)$ had never received the influenza vaccine. Only $10.2 \%$ of students, $19.1 \%$ of patients, and $35.6 \%$ of HCWs reported regular or yearly influenza vaccine uptake. HCWs had the lowest knowledge score about influenza and its vaccine despite high self-reported levels of knowledge. Barriers to vaccinations included lack of information (31\%), fear of adverse effects (29\%), and a perception of not being at risk (23\%). Several factors were independently associated with regular or yearly vaccination uptake including having children (adjusted OR $=3.8 ; 95 \%$ CI 1.2-12.5), a "very good" self-reported level of knowledge (OR $=16.3 ; 95 \%$ CI 1.4-194.2) and being afraid of the consequences of influenza $(\mathrm{OR}=0.2 ; 95 \% \mathrm{CI} 0.1-0.6)$.
\end{abstract}

Conclusion: Adherence rates with regular or yearly vaccination against influenza remain low across all study groups. We were able to identify predictors as well as barriers to vaccination. Future awareness and vaccination campaigns should specifically aim at correcting misconceptions about vaccination, particularly among HCWs, along with addressing the barriers to vaccination. Predictors of vaccination should be integrated in the design of future campaigns.

(c) 2020 Atlantis Press International B.V.

This is an open access article distributed under the CC BY-NC 4.0 license (http://creativecommons.org/licenses/by-nc/4.0/)

\section{INTRODUCTION}

According to the World Health Organization (WHO), influenza epidemics result in 250,000 to 500,000 deaths annually worldwide [1]. In 2009, the H1N1 influenza pandemic posed a worldwide public health threat [2]. Although the WHO International Health Regulations Emergency Committee declared an end to the global pandemic in 2010, the virus itself continues to be transmitted during seasonal outbreaks [3].

Person-to-person transmission of influenza via droplets is common inside and outside health care facilities. Healthcare Workers (HCWs) are at higher risk of occupational acquisition and spreading to healthy staff and vulnerable patients [4,5]. Elderly persons,

"Corresponding author.Email: zk10@aub.edu.lb

Data availability statement: The datasets generated during and/or analyzed during the current study are available from the corresponding author on reasonable request. children, immunocompromised individuals, and pregnant women are at increased risk of infection, leading to several complications and potential deaths [4].

Influenza vaccination decreases the risk of infection in the population, prevents nosocomial infections, decreases morbidity and mortality among patients, and minimizes absenteeism among HCWs [5-7]. It has been estimated that implementing a vaccination program with $100 \%$ adherence for all individuals at risk across the European Union would save 15 million euros spent on primary care visits and approximately 1.6 billion euros due to reduced hospitalizations [8]. Annual Influenza vaccination has been recommended by the US Centers for Disease Control and Prevention (CDC) since the early 1980s (https://www.cdc.gov/vaccines/adults/ index.html). Nevertheless, coverage remains largely inadequate reaching $65 \%$ of the elderly US population [9] and only $35.4 \%$ of the at risk population in the European Union [3]. 
Seasonal Influenza vaccination policies targeting healthcare workers, pregnant women, children above the age of 6 months and elderly and others are implemented in different Eastern Mediterranean countries including Lebanon, Saudi Arabia, Qatar and others. Despite the above policies, vaccination coverage remains low. For instance, vaccination rates in the United Arab Emirates (UAE), Kuwait, and Oman have been reported at $24.7 \%, 67.2 \%$ and $46.4 \%$, respectively [10] while in Turkey, various studies have cited rates ranging from 5.9\% to $27.3 \%$ [11]. Among HCWs in the middle east region, vaccination rates have varied between $12.7 \%$ and $23.1 \%$ in Turkey [12], and a similar study in Saudi Arabia reported a vaccination rate of 41\% [13].

In this study, we aim at identifying factors associated with vaccine use or refusal, in addition to knowledge, perceptions, attitudes and behavior toward influenza vaccination among three different populations: healthy college students, HCWs (physicians and registered nurses), and clinic patients. The rationale behind targeting these three populations was to capture subjects with different and distinct backgrounds, namely young healthy adults with college students, older adults with various health statuses with clinic patients, and professionals with background knowledge on influenza with HCWs. To the best of our knowledge, this study remains among the few studies of its kind to be conducted in the middle east region in recent times and is the first in Lebanon.

\section{MATERIALS AND METHODS}

\subsection{Setting}

We conducted the study at the American University of Beirut (AUB), its affiliated Medical Center (AUBMC) and surrounding hospitals, in Beirut, Lebanon between October 2016 and January 2017. Over 8000 students attend AUB, of whom 51.7\% are male and $48.3 \%$ are female. The facilities included in the study were AUBMC, a 600-bed tertiary referral center for Lebanon and the Middle East, Bikhazi Medical Center, a 150-bed medical-surgical hospital, and the Doctor's Center, an adjacent polyclinic. The study protocol was approved by the AUB Institutional Review Board (IRB).

\subsection{Study Design and Subject Recruitment}

We used a cross-sectional study design with a convenience sample of adults. Several factors affected the determination of sample size and subject recruitment. For a relatively smaller population, a slight reduction in sample size can be performed, albeit the number should remain sufficient for regression data analysis. Adjustments to the size of a sample are also needed to accommodate for comparative analysis of subgroups. The minimum sample size was calculated using a formula for finite population and adjusted for anticipated non-response of $10 \%$, with a target power of 0.80 . Adult participants ( $\geq 18$ years of age) were selected from AUB students, patients at the AUBMC private clinics, and HCWs (physicians and nurses) at the three surveyed medical facilities in order to build our convenience sample. Participants were provided with the surveys and were instructed on how to fill them; to ensure confidentiality, participants dropped the envelope-sealed surveys in a designated box. Recruitment strategies were as follows:

- Students: non-medical students from five different sites on the AUB campus were randomly approached and verbally consented to participate in the study. Target participants on their breaks between courses were approached in university lounges and seating areas where we ensured a suitable atmosphere for students to be able to fill out the questionnaire without distraction.

- Patients: patients who presented to the AUBMC outpatient clinics were randomly approached in five different clinics: dermatology, infectious diseases, endocrinology, rheumatology and gastroenterology. The five different sites and the clinics were selected to cover the largest possible representation of both students and patients. Data collection was performed throughout the 4-month study duration on designated days, once in the morning, and once in the afternoon to ensure comprehensive data sampling. Target participants were approached in waiting areas that are calm and where patients were able to fill out the questionnaire undisturbed.

- HCWs: electronic surveys were sent through LimeSurvey to nurses $(n=50)$ and physicians $(n=50)$ at AUBMC. The email addresses of HCWs were randomly selected by the software from a database owned by the IRB. After three reminders, the response rate was low $(n=24)$. Upon IRB approval and administrative approval from the respective sites, the study was extended to include the two other sites: there, HCWs $(n=28)$ were approached with a paper-based survey in a strategy similar to what was implemented for patients and students. All 28 HCWs accepted to participate in the study, mainly because they were approached individually and face-to-face during their break time rather than using an electronic survey.

\subsection{Survey}

We developed a paper-based self-administered questionnaire that consisted of 32 items divided into four parts: (1) demographic characteristics, (2) knowledge, (3) perceptions and (4) attitudes and behaviors of participants using both open- and close-ended questions. Questions on knowledge were adapted from the CDC webpage "Key Facts" section (https://www.cdc.gov/flu/keyfacts. $\mathrm{htm}$ ), and were used to assess the respondent's general knowledge on influenza, the recommended preventive measures, and vaccination. A score of 1 was given for each correct response. "I don't know" responses were classified as incorrect with a score of 0 . The total maximal score was 6 and the mean score and standard deviation were calculated for every subject category.

\subsection{Statistical Analysis}

All analyses were performed using SPSS16 for Windows (SPSS Inc., Chicago, IL, USA). Chi-squared tests of significance were used to find the association between knowledge, perceptions, attitudes and behavior with different socio-demographic factors. Multivariable regression was used to calculate Odds Ratios (OR) and their 95\% Confidence Intervals (CI) in the measurement of independent associations between the different variables and regular or yearly vaccination. Variables that showed statistical significance on bivariable analysis were included in a multivariable logistic regression model (inclusion cut-off of $p \leq 0.5$ ) with regular or yearly vaccination as a primary outcome (vs. never/sometimes). Variables in the model included age, sex, subject category, marital status, 
having children, and measures of attitudes toward vaccination (preferring to contract influenza over receiving the vaccine, and not being afraid of influenza). We also compared our results to previously collected unpublished data from our medical center records.

\section{RESULTS}

\subsection{Baseline Characteristics}

A total of 327 subjects were approached to participate in the survey and the overall response rate was $75 \%$. The final sample consisted of 247 subjects (100 students, 95 patients, and 52 HCWs) with the response rates for each group being $100 \%, 95 \%$, and $41 \%$, respectively. Table 1 shows the socio-demographic characteristics of the various subgroups. Patients were more likely than other subgroups to selfrate their health as poor or good vs. very good or excellent $(p=0.024)$.

\subsection{History of Influenza Infection and Vaccination}

Of all subjects, 133 (53.8\%) reported ever being diagnosed with influenza; this included the majority of patients $(68.8 \%)$ compared to significantly lower rates in students and HCWs $(47.0 \%$ and $47.8 \%$, respectively; $p=0.005$ ) (Table 2). Although HCWs were significantly more likely to receive the vaccine regularly or yearly $(p<0.001), 117$ subjects $(47.4 \%, n=247)$ reported having never received the vaccine, a substantial proportion of whom were HCWs ( $40 \%$ of all HCWs; $n=52$ ).

\subsection{Knowledge and Perceptions of Influenza and Influenza Vaccine}

The mean knowledge score for all respondent categories was $3.8 \pm 1.6$, with students achieving the highest score $(4.2 \pm 1.1)$ and HCWs the lowest $(3.5 \pm 1.9 ; p=0.014)$. In contrast to the objective score, the self-rated level of knowledge was highest among HCWs, with $40.3 \%$ of subjects in that subgroup reporting a very good to excellent knowledge $(p<0.001)$ (Table 2).

Regarding perceptions about influenza (Table 3), 78\% of all participants perceived influenza as a potentially serious disease, with a majority of students (81\%) and HCWs (100\%) sharing this view compared to a relatively smaller proportion (75\%) of patients $(p=0.002)$. Only $18.6 \%$ of all participants thought vaccination provided a high degree of protection $(>90 \%)$ against influenza, while $29.5 \%$ thought the vaccine efficacy was $<50 \%$. As to vaccine safety, the majority of participants $(85.8 \%)$ considered that the vaccination benefits outweigh the risks.

\subsection{Attitudes and Behaviors toward the Influenza Vaccination}

More patients expressed their desire to receive the vaccine compared to the other groups $(87.8 \%$ vs. $66.0 \%$ and $61.9 \%$ for students and HCWs, respectively; $p=0.001$ ) (Table 4). Students and patients were more willing to get the vaccine if recommended by their health care provider ( $85.1 \%$ and $87.5 \%$, respectively), which was significantly higher compared to HCWs $(57.7 \%$; $p<0.001)$.

Table 1 Socio-demographic and health-related characteristics of the study population

\begin{tabular}{|c|c|c|c|c|}
\hline \multirow{2}{*}{ Variables } & \multicolumn{3}{|c|}{ Subject category $(n=247)$} & \multirow{2}{*}{$p$-value } \\
\hline & College students $(n=100)$ & Outpatients $(n=95)$ & Healthcare workers $(n=52)$ & \\
\hline \multicolumn{5}{|c|}{ Socio-demographic characteristics } \\
\hline Age, in years $($ mean $\pm S D)$ & $20.0 \pm 2.8$ & $43.9 \pm 18.2$ & $38.7 \pm 16.8$ & $<0.001$ \\
\hline Male sex & $38 / 97(39.2)$ & $40 / 90(44.4)$ & $25 / 46(54.3)$ & 0.23 \\
\hline Civil status single & $96 / 99(97.0)$ & $31 / 93(33.3)$ & $24 / 46(52.2)$ & $<0.001$ \\
\hline Has children & $4 / 100(4.0)$ & $58 / 88(65.9)$ & $25 / 46(54.3)$ & $<0.001$ \\
\hline No. of children (mean \pm SD) & $0.1 \pm 0.5$ & $1.9 \pm 1.6$ & $1.3 \pm 1.4$ & $<0.001$ \\
\hline Monthly household income & & & & 0.06 \\
\hline 400 to $<800$ USD & $7 / 83(8.4)$ & $15 / 80(18.8)$ & $1 / 43(2.3)$ & \\
\hline 800 to 1600 USD & $23 / 83(27.7)$ & $23 / 80(28.8)$ & $14 / 43(32.6)$ & \\
\hline$>1600$ USD & $53 / 83(63.9)$ & $42 / 80(52.5)$ & $28 / 43(65.1)$ & \\
\hline \multicolumn{5}{|c|}{ Behavioral and health-related characteristics } \\
\hline Smoking & & & & 0.02 \\
\hline Never/occasionally & $88 / 100(88.0)$ & $69 / 94(73.4)$ & $40 / 46(87.0)$ & \\
\hline Frequently/regularly & $12 / 100(12.0)$ & $25 / 94(26.6)$ & $6 / 46(13.0)$ & \\
\hline Alcohol & & & & 0.31 \\
\hline Never/occasionally & $85 / 100(85.0)$ & $83 / 93(89.2)$ & $43 / 46(93.5)$ & \\
\hline Frequently/regularly & $15 / 100(15.0)$ & $10 / 93(10.8)$ & $3 / 46(6.5)$ & \\
\hline Exercise & & & & 0.48 \\
\hline Never/occasionally & $52 / 100(52.0)$ & $57 / 94(60.6)$ & $25 / 45(55.6)$ & \\
\hline Frequently/regularly & $48 / 100(48.0)$ & $37 / 95(38.9)$ & $20 / 45(44.4)$ & \\
\hline Self-rated health & & & & 0.02 \\
\hline Poor & $2 / 100(2.0)$ & $7 / 94(7.4)$ & $0(0.0)$ & \\
\hline Good & $48 / 100(48.0)$ & $57 / 94(60.6)$ & $24 / 44(54.5)$ & \\
\hline Very good/excellent & $50 / 100(50.0)$ & 30/94 (31.9) & $20 / 44(45.5)$ & \\
\hline Comorbid conditions ${ }^{*}$ & 7/98 (7.1) & 18/94 (19.1) & $6 / 50(12.0)$ & 0.04 \\
\hline
\end{tabular}

‘Lung, heart, or kidney disease. All numbers indicate $n / N(\%)$ unless otherwise specified. USD, US dollars. 
Table 2 Personal experience with and knowledge about influenza virus and vaccination in the study population

\begin{tabular}{|c|c|c|c|c|}
\hline \multirow[b]{2}{*}{ Variables } & \multicolumn{3}{|c|}{ Subject category } & \multirow[b]{2}{*}{$p$-value } \\
\hline & $\begin{array}{l}\text { College students } \\
\qquad(n=100)\end{array}$ & $\begin{array}{l}\text { Outpatients } \\
\qquad(n=95)\end{array}$ & $\begin{array}{l}\text { Healthcare workers } \\
\qquad(n=52)\end{array}$ & \\
\hline \multicolumn{5}{|l|}{ Personal experience with influenza virus and vaccination } \\
\hline Ever had influenza & $47 / 100(47.0)$ & $64 / 93(68.8)$ & $22 / 46(47.8)$ & 0.005 \\
\hline Reported current likelihood of contracting influenza & & & & 0.33 \\
\hline Unlikely & $25 / 100(25.0)$ & $28 / 95(29.5)$ & $19 / 52(36.5)$ & \\
\hline Likely & $75 / 100(75.0)$ & $67 / 95(70.5)$ & $33 / 52(63.5)$ & \\
\hline Receipt of vaccine & & & & $<0.001$ \\
\hline Never & 41/98 (41.8) & 58/94 (61.7) & $18 / 45(40.0)$ & \\
\hline Sometimes & $47 / 98(48.0)$ & $18 / 94(19.1)$ & $11 / 45(24.4)$ & \\
\hline Regularly/yearly & $10 / 98(10.2)$ & $18 / 94(19.1)$ & $16 / 45(35.6)$ & \\
\hline \multicolumn{5}{|l|}{ Knowledge about influenza virus and vaccination } \\
\hline Knowledge score* $($ mean \pm SD) & $4.2 \pm 1.1$ & $3.8 \pm 1.4$ & $3.5 \pm 1.9$ & 0.01 \\
\hline Self-rated knowledge about influenza & & & & $<0.001$ \\
\hline Poor & $25 / 100(25.0)$ & $19 / 95(20.0)$ & $11 / 52(21.2)$ & \\
\hline Good & $72 / 100(72.0)$ & $64 / 95(67.4)$ & $20 / 52(38.5)$ & \\
\hline Very good & 0 & $6 / 95(6.3)$ & $15 / 52(28.8)$ & \\
\hline Excellent & $3 / 100(3.0)$ & $6 / 95(6.3)$ & $6 / 52(11.5)$ & \\
\hline Source of health information & & & & 0.70 \\
\hline Physician or specialized media ${ }^{* *}$ & $60 / 96(62.5)$ & $61 / 90(67.8)$ & $26 / 42(61.9)$ & \\
\hline Family, friends, or general media & $36 / 96(37.5)$ & $29 / 90(32.2)$ & $16 / 42(38.1)$ & \\
\hline
\end{tabular}

"Knowledge score out of 6 . "Specialized media include scientific channels, websites, or magazines. All numbers indicate $n / N(\%)$ unless otherwise specified.

Table 3 Perceptions about the influenza virus and vaccine in the study population

\begin{tabular}{lccc}
\hline & \multicolumn{3}{c}{ Subject category } \\
\cline { 2 - 3 } Variables & $\begin{array}{c}\text { College students } \\
(\boldsymbol{n}=\mathbf{1 0 0})\end{array}$ & $\begin{array}{c}\text { Outpatients } \\
(\boldsymbol{n = 9 5 )}\end{array}$ & $\begin{array}{c}\text { Healthcare workers } \\
(\boldsymbol{n}=\mathbf{5 2})\end{array}$ \\
\hline Influenza can be a serious disease & $81 / 100(81.0)$ & $69 / 92(75.0)$ & $42 / 42(100)$ \\
Influenza vaccine provides...\% immunization against influenza & & & \\
$\quad<50 \%$ & $22 / 100(22.0)$ & $29 / 95(30.5)$ & $22 / 52(42.3)$ \\
$50-90 \%$ & $46 / 100(46.0)$ & $42 / 95(44.0)$ & $17 / 52(32.7)$ \\
$91-100 \%$ & $15 / 100(15.0)$ & $7 / 95(7.4)$ & $2 / 52(3.8)$ \\
$\quad$ Don't know & $17 / 100(17.0)$ & $17 / 95(17.9)$ & $11 / 52(21.2)$ \\
Influenza vaccine is... & & & \\
$\quad$ Very safe & $29 / 100(29.0)$ & $27 / 95(28.4)$ & $19 / 52(36.5)$ \\
$\quad$ Somewhat safe & $48 / 100(48.0)$ & $53 / 95(55.8)$ & $24 / 52(46.2)$ \\
$\quad$ Not safe & $6 / 100(6.0)$ & $5 / 95(5.3)$ & $4 / 52(7.7)$ \\
$\quad$ Don't know & $17 / 100(17.0)$ & $10 / 95(10.5)$ & $5 / 52(9.6)$ \\
Benefits from influenza vaccine outweigh risks & $83 / 100(83.0)$ & $82 / 95(86.3)$ & $47 / 52(90.4)$ \\
\hline
\end{tabular}

All numbers indicate $n / N(\%)$ unless otherwise specified.

Around $65 \%$ of patients and HCWs $(n=146)$ were willing to vaccinate their children against influenza. Also, patients were more willing than HCWs to vaccinate their children $(72.3 \%$ vs. $51.9 \%$; $p=0.01)$. The most cited reason for refusing vaccination was lack of information about the vaccine (31\%), fear of potential adverse effects (29\%), and the thought of not being at risk (23\%). There was no difference in the cited reasons across the three study populations.

\subsection{Determinants of Regular or Yearly Vaccination Uptake}

Approximately, 52\% of the study population reported regular or yearly receipt of influenza vaccine, and this was less prevalent among students compared to patients and HCWs $(p=0.001)$. The results of the bivariable analysis are shown in Table 5: a higher mean age $(p<0.001)$, being married/widowed $(\mathrm{OR}=2.5 ; p=$ $0.006)$, having children $(\mathrm{OR}=3.7 ; p<0.001)$, and physician recommendation of the vaccine $(\mathrm{OR}=7.1 ; p<0.001)$ were all associated with regular or yearly influenza vaccination. While a good self-reported knowledge about flu symptoms and having a positive attitude toward vaccination showed a positive association, having a higher objective knowledge score was not associated with regular or yearly vaccination uptake $(p=0.63)$.

The multivariable logistic regression model revealed three independent predictors of regular or yearly influenza vaccination: having children (adjusted OR $=3.8$; 95\% CI 1.2-12.5) and having a "very good" self-reported knowledge of the influenza symptoms 
Table 4 Attitudes and behaviors toward influenza vaccination

\begin{tabular}{|c|c|c|c|c|}
\hline \multirow[b]{2}{*}{ Variables } & \multicolumn{3}{|c|}{ Subject category } & \multirow[b]{2}{*}{$p$-value } \\
\hline & $\begin{array}{l}\text { College students } \\
\qquad(n=100)\end{array}$ & $\begin{array}{l}\text { Outpatients } \\
\qquad(n=95)\end{array}$ & $\begin{array}{c}\text { Healthcare } \\
\text { workers }(n=52)\end{array}$ & \\
\hline \multicolumn{5}{|l|}{ (A) Attitudes } \\
\hline I feel that getting a flu shot is a wise thing to do & $74 / 100(74.0)$ & $75 / 95(78.9)$ & $32 / 52(61.5)$ & 0.07 \\
\hline I am willing to get the flu shot & $64 / 97(66.0)$ & $72 / 82(87.8)$ & $26 / 42(61.9)$ & 0.001 \\
\hline I would rather get the flu shot than get the flu & $75 / 99(75.8)$ & $69 / 80(86.3)$ & $36 / 42(85.7)$ & 0.15 \\
\hline I think the flu vaccine causes influenza & $22 / 100(22.0)$ & $12 / 95(12.6)$ & $11 / 52(21.2)$ & 0.20 \\
\hline I am not afraid of influenza & $45 / 100(45.0)$ & $45 / 95(47.4)$ & $26 / 52(50.0)$ & 0.84 \\
\hline \multicolumn{5}{|l|}{ (B) Behaviors } \\
\hline I would recommend vaccination against the flu & $68 / 100(68.0)$ & $66 / 95(69.5)$ & $32 / 52(61.5)$ & 0.60 \\
\hline After the last flu pandemic, I am more likely to recommend vaccination to my patients & N/A & $\mathrm{N} / \mathrm{A}$ & $32 / 52(61.5)$ & NA \\
\hline I ask my health care provider for the flu shot & $55 / 100(55.0)$ & $49 / 95(51.6)$ & N/A & 0.63 \\
\hline I would get a flu shot if my health care provider recommended it & $80 / 94(85.1)$ & $70 / 80(87.5)$ & $30 / 52(57.7)$ & $<0.001$ \\
\hline I would vaccinate my children against influenza & $\mathrm{N} / \mathrm{A}$ & $68 / 94(72.3)$ & $27 / 52(51.9)$ & 0.01 \\
\hline
\end{tabular}

All numbers indicate $n / N(\%)$ unless otherwise specified. N/A, not applicable.

Table 5 Bivariable analysis of potential determinants of regular or yearly influenza vaccination

\begin{tabular}{|c|c|c|c|c|}
\hline \multirow{2}{*}{ Variables } & \multicolumn{2}{|c|}{ Influenza vaccine uptake } & \multirow{2}{*}{$\begin{array}{l}\text { Unadjusted } \\
\text { odds ratio }\end{array}$} & \multirow{2}{*}{$p$-value } \\
\hline & Sometimes/never $(n=193)$ & Regularly/yearly $(n=44)$ & & \\
\hline Subject category & & & & 0.001 \\
\hline College students & $88 / 193(45.6)$ & $10 / 44(22.7)$ & & \\
\hline Outpatients & $76 / 193(39.4)$ & $18 / 44(40.9)$ & & \\
\hline Healthcare workers & $29 / 193(15.0)$ & $16 / 44(36.4)$ & & \\
\hline Age & $30.8 \pm 15.7$ & $40.8 \pm 19.3$ & & $<0.001$ \\
\hline Marital status married/widowed & $62 / 191(32.5)$ & $24 / 44(54.5)$ & 2.5 & 0.006 \\
\hline Having children & $59 / 187(31.6)$ & $27 / 43(62.8)$ & 3.7 & $<0.001$ \\
\hline Average number of children & $0.9 \pm 1.5$ & $1.6 \pm 1.4$ & & 0.003 \\
\hline Knowledge score ${ }^{*}$ & $4.0 \pm 1.2$ & $4.1 \pm 1.3$ & & 0.63 \\
\hline Self-rated knowledge about influenza & & & & 0.01 \\
\hline Poor & $41 / 193(21.2)$ & $4 / 44(9.1)$ & & \\
\hline Good & $129 / 193(66.8)$ & $27 / 44(61.4)$ & & \\
\hline Very good & $13 / 193(6.7)$ & $8 / 44(18.2)$ & & \\
\hline Excellent & $10 / 193(5.2)$ & $5 / 44(11.4)$ & & \\
\hline Vaccine recommended by physician & $65 / 148(43.9)$ & $21 / 25(84.0)$ & 7.1 & $<0.001$ \\
\hline I am willing to get the flu shot & $123 / 178(69.1)$ & $37 / 41(90.2)$ & 4.2 & 0.006 \\
\hline I would rather get the flu shot than the flu & $139 / 178(78.0)$ & $39 / 41(95.1)$ & 5.6 & 0.01 \\
\hline I am not afraid of influenza & $102 / 193(52.8)$ & $13 / 44(29.5)$ & 2.7 & 0.005 \\
\hline I would recommend vaccination against the flu & $126 / 193(65.3)$ & $38 / 44(86.4)$ & 3.3 & 0.006 \\
\hline In the future, I would like to receive vaccination & $126 / 193(65.3)$ & $37 / 44(84.1)$ & 2.8 & 0.01 \\
\hline I ask my health care provider for the flu vaccine & $81 / 164(49.4)$ & $21 / 28(75.0)$ & 3.0 & 0.01 \\
\hline
\end{tabular}

*Knowledge score out of 6 . All numbers indicate $n / N(\%)$ unless otherwise specified.

(adjusted OR $=16.3$; 95\% CI 1.4-194.2). Not being afraid of the flu was negatively correlated with receipt of the vaccine (adjusted $\mathrm{OR}=0.2 ; 95 \%$ CI $0.1-0.6$ ).

\section{DISCUSSION}

In this cross-sectional study, self-reported vaccination was low among patients, students and HCWs in Lebanon, reaching no more that $36 \%$ among HCWs, despite agreement about the seriousness of influenza and a high perceived likelihood of contracting the infection. This finding is validated by the University Health Services data reporting a vaccination uptake of $38.5 \%$ and $33.4 \%$ in 2016 and 2017, respectively. Compared to neighboring countries, our HCWs vaccination uptake falls within regional data and ranks lower than Kuwait (67.2\%), Oman (46.4\%) and Saudi Arabia (41\%) $[10,13]$. In contrast, they supersede those reported in Turkey (12.7-23.1\%) and the UAE (24.7\%) [10-12]. HCWs overestimate their knowledge about influenza while scoring lowest on the objective knowledge component. Low levels of knowledge about the vaccine were previously shown to cause lower vaccination rates in nurses compared to physicians $[5,14,15]$. The low rates of adherence with vaccination among physicians may be traced back to a tradition of neglecting their own health in favor of their many professional and personal obligations [16]. The relatively small number of HCWs in this study does not allow for subgroup analysis to delineate differences between nurses and physicians. A recent study in Pakistan showed low vaccination rates among physicians against 
influenza (8.84\%), with lack of awareness about the vaccine and the recommendations being the major obstacles for adherence [17].

Vaccination uptake was lowest among students (10.2\%), with a significantly lower likelihood of regular vaccination $(p=0.001)$, despite responses of high likelihood of contracting influenza (75.0\%). Similar low rates of influenza vaccination among students were reported in studies from European countries [18,19]: students could perceive themselves as healthy individuals susceptible to influenza but at lesser risk for its complications. They also visit healthcare providers less frequently. In fact, people who view themselves as healthy are usually less likely to take the vaccine, while those with poorer self-rated health and higher co-morbidities have a higher vaccination rate [20].

Previous studies have shown that among HCWs, adherence to vaccination manifested in their recommendation to patients $[5,15,21,22]$. In our cohort, HCWs demonstrated the lowest objective knowledge scores despite reporting a significantly higher level of self-rated knowledge. These results are supported by another study showing that while $85 \%$ of nurses believed they received the necessary information to make an adequate decision about influenza vaccination, only $9.6 \%$ correctly answered knowledge questions [23]. During the last $\mathrm{H} 1 \mathrm{N1}$ pandemic (2009-2010), adequate basic knowledge of HCWs about the vaccine was strongly associated with recommending it to patients [24] and correlated with higher rates of vaccination in older community-dwelling adults [25].

A distrust regarding the vaccine is prevalent in our study with doubts regarding its effectiveness and ambivalence as to its safety. A third of participants stated fear of potential adverse effects as a deterrent from vaccination. Many studies have recently investigated adverse effects of the seasonal influenza vaccine, with concerns being raised regarding potential effects on pregnancy [26-28], neurological diseases [29-31], and potential solid organ transplantation rejection [32]. These studies have not been conclusive enough, and the CDC has not modified its recommendation of yearly vaccination. Considering these doubts and misconceptions, HCWs could act as a source of accurate information regarding the vaccine efficacy and safety. In fact, $77 \%$ of participants stated their willingness to take the vaccine if recommended by a physician. Studies in the United Kingdom, France, Germany, and Mexico showed that HCWs and the general population alike consulted their practitioner before obtaining the free-of-charge influenza vaccine $[19,24,33]$. The lack of recommendation by a physician was further shown to significantly hinder immunization $[24,33,34]$.

Unlike the objective knowledge score, a self-reported knowledge of "very good" was the strongest independent predictor of vaccination $(\mathrm{OR}=16.3$; 95\% CI 1.4-194.2). Therefore, perceived rather than objective knowledge seems to drive participants to vaccination. Efforts should however be centered on bridging the gap between the self-reported knowledge level and actual knowledge among HCWs.

The positive association between having children and vaccine uptake is a new finding that has not been addressed in previous studies. It can be attributed to the sense of responsibility individuals feel toward their children. This aspect could be further exploited in awareness campaigns using "protect your children" as a message.
Overall, having a positive attitude toward vaccination was associated with increased uptake of regular or yearly vaccination. Patients who reported not being afraid of the flu were $80 \%$ less likely to receive the vaccine, and this was supported by other studies in healthy adults and HCWs $[21,24]$. Thus, clarifying the potential severity of influenza should be a key element in future vaccination campaigns. In a prior assessment of influenza vaccine uptake at our institution, having a positive attitude consistently predicted regular vaccination in individual sub-groups and across all participants $(\mathrm{OR}=38.7$; 95\% CI 38.7-38.8). These determinants have been echoed in regional studies performed in Turkey and several Arab countries mentioned above [10-13], thus highlighting a possible cultural context to be considered while building future campaigns.

In a recent study by Yeung et al. [35], a systematic review of 23 articles assessed factors associated with yearly influenza vaccination uptake. Perceptions about vaccine efficacy (OR 2.7-10.5), safety and adverse events (OR 10.5) were more influential than the level of knowledge itself. Advice from doctors was also a key predictive factor of vaccination. Moreover, in the US, individuals with higher education, better income, and insurance coverage have higher vaccination rates $[17,36]$.

Based on our findings, we propose a model for a locally effective influenza vaccination awareness campaign using the framework of the Health Belief Model (HBM) [37]. Such an approach was undertaken in a Chinese study to provide a description of the high-risk sexual behavior in men who have sex with men [38]. The model presents a framework of constructs that, when adapted to the influenza disease and vaccination, consists of the following elements: (i) perceived susceptibility (increasing awareness of risk); (ii) perceived severity (stressing potential complications); (iii) perceived benefits (protection of self and others, especially children); (iv) perceived barriers (accurate depiction of vaccine adverse events and effectiveness); and (v) cues to action (using frequent reminders). The model is based on the assumption that a person's belief in a personal threat of an illness or disease together with his/her belief in the effectiveness of the recommended health behavior will predict the likelihood the person will adapt the behavior. We believe the HBM can help explore or identify approaches that may be used to increase adherence to vaccination. Even though multiple factors in our surveys were shown to correlate with vaccination, we believe that these factors require a further exploration through descriptive qualitative study designs using focus groups and in-depth interviews.

The current vaccination uptake is higher compared to our 2010 findings but remains significantly lower than US data $(68.5 \%$ for HCWs and $40 \%$ for the general population) and data from neighboring countries, namely Kuwait, Oman and Saudi Arabia [10]. These rates are higher than earlier findings whereby adherence rates were $25.5 \%$ and $7.1 \%$ for HCWs and students, respectively (data from the University Health Services at AUB).

Whether vaccination should be mandatory to all HCWs remains debatable: among 97 facilities in the US, there was a $25.5 \%$ increase in vaccination rate between pre-mandates and post-mandates in hospitals with consequences for non-adherence, versus $15 \%$ in hospitals without consequences [39]. Consequences ranged from wearing a mask during the influenza season to termination. Currently, AUBMC adopts a strategy of strongly encouraging influenza vaccination rather than making it compulsory. 
Our study is limited by the small sample size and low response rates from HCWs. This raises two important potential biases: the under coverage of the population, and the non-response bias, especially given the low response rate from HCWs. Participants self-reported their vaccination status, and one cannot rule out the possibility of over reporting the vaccination uptake as a manifestation of social desirability. Our population consists of a convenience sample that may not be representative of the larger population of students, patients and HCWs at our institution. Therefore, large multicenter and population-based studies are needed to produce generalizable data. Lastly, and despite the measures taken to ensure confidentiality, bias based on social desirability and conformity especially amongst students cannot be ruled out given the study design.

\section{CONCLUSION}

Low uptake of influenza vaccination remains a global problem. In this study, we identified three major independent predictors of regular or yearly vaccination uptake: having children, having a "very good" self-reported knowledge of influenza, and being afraid of contracting the flu virus. These, along with socio-demographic background should be considered to develop an efficient national influenza vaccination campaign. Based on our findings we have presented a model for a locally effective campaign based on the HBM framework.

\section{CONFLICTS OF INTEREST}

The authors declare they have no conflicts of interest.

\section{AUTHORS' CONTRIBUTION}

Project inception: NE, HH and ZK. Data collection: KC, JE, NE, $\mathrm{HH}, \mathrm{NS}, \mathrm{MY}$ and AR. Statistical analysis: KC, SA, NE and ZK. Write-up: KC, NE, HH and ZK. Manuscript revision: SK and ZK.

\section{FUNDING}

This research did not receive any specific grant from funding agencies in the public, commercial, or not-for-profit sectors.

\section{ACKNOWLEDGMENT}

The authors would like to acknowledge Dr. Jamil Kazma for his help in formatting and finalizing the manuscript.

\section{REFERENCES}

[1] Thompson WW, Weintraub E, Dhankhar P, Cheng PY, Brammer L, Meltzer MI, et al. Estimates of US influenzaassociated deaths made using four different methods. Influenza Other Respir Viruses 2009;3;37-49.

[2] Arda B, Durusoy R, Yamazhan T, Sipahi OR, Taşbakan M, Pullukçu H, et al. Did the pandemic have an impact on influenza vaccination attitude? a survey among health care workers. BMC Infect Dis 2011;11;87.

[3] Ryan J, Zoellner Y, Gradl B, Palache B, Medema J. Establishing the health and economic impact of influenza vaccination within the European Union 25 countries. Vaccine 2006;24;6812-22.

[4] Kruy SL, Buisson Y, Buchy P. [Asia: avian influenza H5N1]. Bull Soc Pathol Exot 2008;101;238-42 (in French).

[5] Martinello RA, Jones L, Topal JE. Correlation between healthcare workers' knowledge of influenza vaccine and vaccine receipt. Infect Control Hosp Epidemiol 2003;24;845-7.

[6] Hollmeyer HG, Hayden F, Poland G, Buchholz U. Influenza vaccination of health care workers in hospitals-a review of studies on attitudes and predictors. Vaccine 2009;27;3935-44.

[7] Torun SD, Torun F. Vaccination against pandemic influenza A/H1N1 among healthcare workers and reasons for refusing vaccination in Istanbul in last pandemic alert phase. Vaccine 2010;28;5703-10.

[8] Uhart M, Bricout H, Clay E, Largeron N. Public health and economic impact of seasonal influenza vaccination with quadrivalent influenza vaccines compared to trivalent influenza vaccines in Europe. Hum Vaccin Immunother 2016;12;2259-68.

[9] Simonsen L, Taylor RJ, Viboud C, Miller MA, Jackson LA. Mortality benefits of influenza vaccination in elderly people: an ongoing controversy. Lancet Infect Dis 2007;7;658-66.

[10] Abu-Gharbieh E, Fahmy S, Rasool BA, Khan S. Influenza vaccination: healthcare workers attitude in three Middle East countries. Int J Med Sci 2010;7;319-25.

[11] Ciblak MA, Platformu G. Influenza vaccination in Turkey: prevalence of risk groups, current vaccination status, factors influencing vaccine uptake and steps taken to increase vaccination rate. Vaccine 2013;31;518-23.

[12] Akan H, Yavuz E, Yayla ME, Külbay H, Kaspar EÇ, Zahmacıŏglu $\mathrm{O}$, et al. Factors affecting uptake of influenza vaccination among family physicians. Vaccine 2016;34;1712-18.

[13] Al-Tawfiq JA. Willingness of health care workers of various nationalities to accept H1N1 (2009) pandemic influenza A vaccination. Ann Saudi Med 2012;32;64-7.

[14] Esposito S, Bosis S, Pelucchi C, Tremolati E, Sabatini C, Semino M, et al. Influenza vaccination among healthcare workers in a multidisciplinary University hospital in Italy. BMC Public Health $2008 ; 8 ; 422$.

[15] Qureshi A, Hughes NJM, Murphy E, Primrose WR. Factors influencing uptake of influenza vaccination among hospital-based health care workers. Occup Med (Lond) 2004;54;197-201.

[16] Tyzuk K. Physician health: a review of lifestyle behaviors and preventive health care among physicians. BC Med J 2012;54;419-23.

[17] Ali I, Ijaz M, Rehman IU, Rahim A, Ata H. Knowledge, attitude, awareness, and barriers toward influenza vaccination among medical doctors at tertiary care health settings in peshawar, Pakistan-a cross-sectional study. Front Public Health 2018;6;173.

[18] Blank PR, Schwenkglenks M, Szucs TD. Vaccination coverage rates in eleven European countries during two consecutive influenza seasons. J Infect 2009;58;446-58.

[19] Endrich MM, Blank PR, Szucs TD. Influenza vaccination uptake and socioeconomic determinants in 11 European countries. Vaccine 2009;27;4018-24.

[20] Ward L, Draper J. A review of the factors involved in older people's decision making with regard to influenza vaccination: a literature review. J Clin Nurs 2008;17;5-16. 
[21] Chapman GB, Coups EJ. Predictors of influenza vaccine acceptance among healthy adults. Prev Med 1999;29;249-62.

[22] Hofmann F, Ferracin C, Marsh G, Dumas R. Influenza vaccination of healthcare workers: a literature review of attitudes and beliefs. Infection 2006;34;142-7.

[23] Ofstead CL, Tucker SJ, Beebe TJ, Poland GA. Influenza vaccination among registered nurses: information receipt, knowledge, and decision-making at an institution with a multifaceted educational program. Infect Control Hosp Epidemiol 2008;29; 99-106.

[24] Bovier PA, Chamot E, Bouvier Gallacchi M, Loutan L. Importance of patients' perceptions and general practitioners' recommendations in understanding missed opportunities for immunisations in Swiss adults. Vaccine 2001;19;4760-7.

[25] Nowalk MP, Zimmerman RK, Shen S, Jewell IK, Raymund M. Barriers to pneumococcal and influenza vaccination in older community-dwelling adults (2000-2001). J Am Geriatr Soc 2004;52;25-30.

[26] Kharbanda EO, Vazquez-Benitez G, Lipkind H, Naleway A, Lee G, Nordin JD, et al. Inactivated influenza vaccine during pregnancy and risks for adverse obstetric events. Obstet Gynecol 2013;122;659-67.

[27] Ludvigsson JF, Zugna D, Cnattingius S, Richiardi L, Ekbom A, Örtqvist $\AA$, et al. Influenza H1N1 vaccination and adverse pregnancy outcome. Eur J Epidemiol 2013;28;579-88.

[28] Richards JL, Hansen C, Bredfeldt C, Bednarczyk RA, Steinhoff MC, Adjaye-Gbewonyo D, et al. Neonatal outcomes after antenatal influenza immunization during the $2009 \mathrm{H} 1 \mathrm{~N} 1$ influenza pandemic: impact on preterm birth, birth weight, and small for gestational age birth. Clin Infect Dis 2013;56;1216-22.

[29] Barker CI, Snape MD. Pandemic influenza A H1N1 vaccines and narcolepsy: vaccine safety surveillance in action. Lancet Infect Dis $2014 ; 14 ; 227-38$.
[30] Haber P, DeStefano F, Angulo FJ, Iskander J, Shadomy SV, Weintraub E, et al. Guillain-Barré syndrome following influenza vaccination. JAMA 2004;292;2478-81.

[31] Salmon DA, Proschan M, Forshee R, Gargiullo P, Bleser W, Burwen DR, et al. Association between Guillain-Barré syndrome and influenza A (H1N1) 2009 monovalent inactivated vaccines in the USA: a meta-analysis. Lancet 2013;381;1461-8.

[32] Cohet C, Haguinet F, Dos Santos G, Webb D, Logie J, Ferreira GL, et al. Effect of the adjuvanted (AS03) A/H1N1 2009 pandemic influenza vaccine on the risk of rejection in solid organ transplant recipients in England: a self-controlled case series. BMJ Open 2016;6;e009264.

[33] Telford R, Rogers A. What influences elderly peoples' decisions about whether to accept the influenza vaccination? A qualitative study. Health Educ Res 2003;18;743-53.

[34] Weinstein ND, Kwitel A, McCaul KD, Magnan RE, Gerrard M, Gibbons FX. Risk perceptions: assessment and relationship to influenza vaccination. Health Psychol 2007;26;146-51.

[35] Yeung MPS, Lam FLY, Coker R. Factors associated with the uptake of seasonal influenza vaccination in adults: a systematic review. J Public Health (Oxf) 2016;38;746-53.

[36] Chi RC, Neuzil KM. The association of sociodemographic factors and patient attitudes on influenza vaccination rates in older persons. Am J Med Sci 2004;327;113-17.

[37] Janz NK, Becker MH. The Health Belief Model: a decade later. Health Educ Q 1984;11;1-47.

[38] Li X, Lei Y, Wang H, He G, Williams AB. The Health Belief Model: a qualitative study to understand high-risk sexual behavior in chinese men who have sex with men. J Assoc Nurses AIDS Care 2016;27;66-76.

[39] Nowalk MP, Lin CJ, Raymund M, Bialor J, Zimmerman RK. Impact of hospital policies on health care workers' influenza vaccination rates. Am J Infect Control 2013;41;697-701. 